\title{
Pointillist melanotic macules: a new clinical entity?
}

\author{
Cheng Tan', Wen-Yuan Zhu \\ ${ }^{1}$ Department of Dermatology, First Affiliated Hospital of Nanjing University of Traditional Chinese Medicine, Nanjing, China \\ Head: Prof. Zhong-Sheng Min MD \\ 2Department of Dermatology, First Affiliated Hospital of Nanjing Medical University, Nanjing, China \\ Head: Prof. Dan Luo MD
}

Postep Derm Alergol 2012; XXIX, 6: 475-479

DOI: 10.5114/pdia.2012.32398

\begin{abstract}
A Chinese male and his father both presented disseminated, variably-sized and pigmented spots as well as stellate macules in their adulthood. No signs indicated a systemic involvement after extensive evaluation. Skin examination revealed that most macules were stellate or claw-like that differ from each other in shape and size. However, as a whole, these discrete lesions were interspersed regularly. Pathological changes included remarkable basilar layer hyperpigmentation (black eye line sign) without proliferation of the melanocytes. Melanophages were noted in the dermis. We proposed the diagnosis of pointillist melanotic macules, and believe this is a new clinical entity.
\end{abstract}

Key words: hyperpigmentation, melanotic, macules, hypermelanosis.

\section{Introduction}

Categorizing pigmentary skin disorders according to the morphology of the lesions is an efficient way to establish an appropriate diagnosis. A perfect example of this is reticular hypermelanosis on the extremities that can easily lead to the diagnosis of reticulate acropigmentation of kitamura. We, dermatologists, are usually more familiar with round, oval or linear lesions than others. If stellate black macules are present, is there any diagnosis we can still choose? We are now in such a diagnostic dilemma in two patients, who manifested variably-sized, pigmented spots and stellate macules. This sight was strongly reminiscent of the painting technique of a pointillist, which accentuates the application of small dots and brush strokes to create the impression of a wide selection of other colors and optical blending. We suggested a diagnosis of pointillist melanotic macules to the following patients.

\section{Case reports}

\section{Case 1}

A 28-year-old Chinese male was referred to us for the evaluation of numerous black macules for 4 years. The lesions appeared first on both upper arms, extending then to the neck and finally spread to the whole trunk. There was no prior inflammation or bullae formation. No itching or other abnormal sensations accompanied. He denied any abnormal medication, nor did he expose himself to culprit chemicals or metals that might be causative for a disseminated hyperpigmentation. His general health condition was excellent; ophthalmological, neurological and other system examinations retrieved noncontributory findings. Skin examination showed thousands of pigmented macules disseminated on the trunk, neck and extremities. The chest is the most densely involved. Face and mucous were all spared. A closer inspection revealed that these melanotic lesions consisted of uniformly-intermingled spots and stellate (or claw-like) macules, and simultaneously, a black 'pointillist painting' burst upon our minds: the macules were evenly pigmented in such an impressive way of distinct, stellate or claw-like borders. The spots were uniformly pin-sized, oval or round, whereas the bizarre macules varied ranging from $0.1 \mathrm{~cm}$ to $3 \mathrm{~cm}$ in diameter (Figures $1 \mathrm{~A}$ and $\mathrm{B})$. The texture of the skin was normal. White macules and keratotic lesions were not noted. Hair, nail, sweat gland and sebum gland were all normal. Potassium hydroxide examination was negative for hyphae. Skin biopsy showed that the epidermis was otherwise normal except remarkable basilar hyperpigmentation. No junctional nests of melanocytes or melanocytic hyperplasia were noted. Rete ridges were mildly elongated. The dermis was almost normal except foci melanophages in the papillary dermis (Figures $2 \mathrm{~A}$ and $\mathrm{B}$ ). The rest of the examination revealed a well-appearing normal blood test, negative

Address for correspondence: Cheng Tan MD, Department of Dermatology, First Affiliated Hospital of Nanjing University of Traditional Chinese Medicine, 155 Hanzhong Road, 210029 Nanjing, China, phone: +86 13515102233, e-mail: tancheng@medmail.com.cn 
A

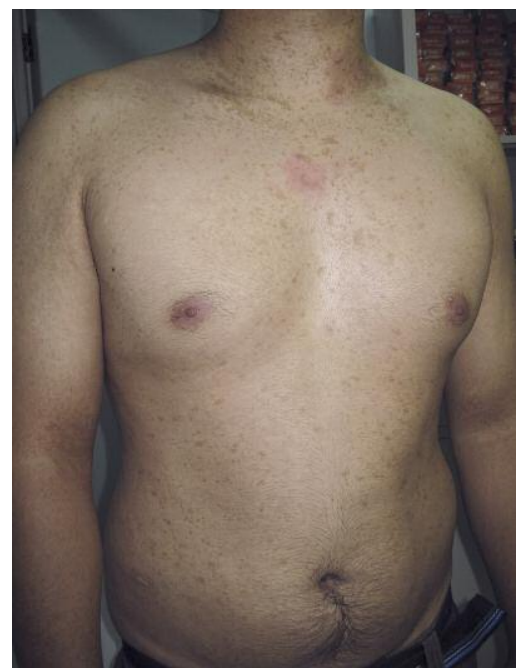

B

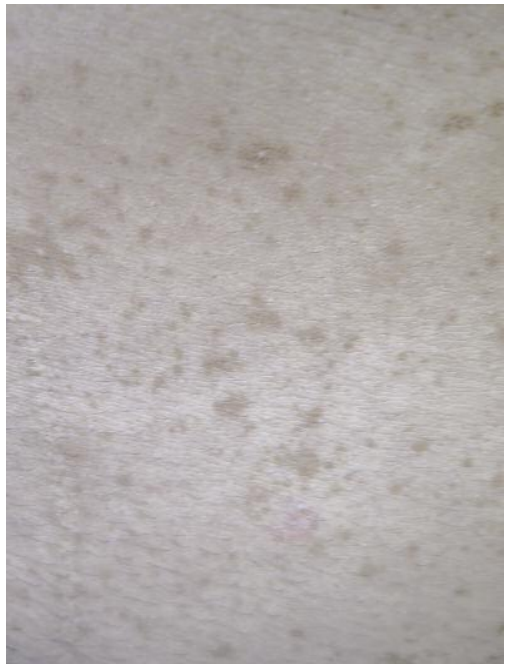

Figure 1. A - Thousands of pigmented macules disseminated on the trunk, neck and extremities. The chest is the most densely involved. B - A closer inspection show uniformly-intermingled spots and stellate (or claw-like) macules, like a black 'pointillist body painting'. The macules were evenly dark colored in such an impressive way of distinct, stellate or clawlike borders. The spots were uniformly pinsized, oval or round, whereas the bizarre macules varied ranges from $0.1 \mathrm{~cm}$ to several centimeters in diameter

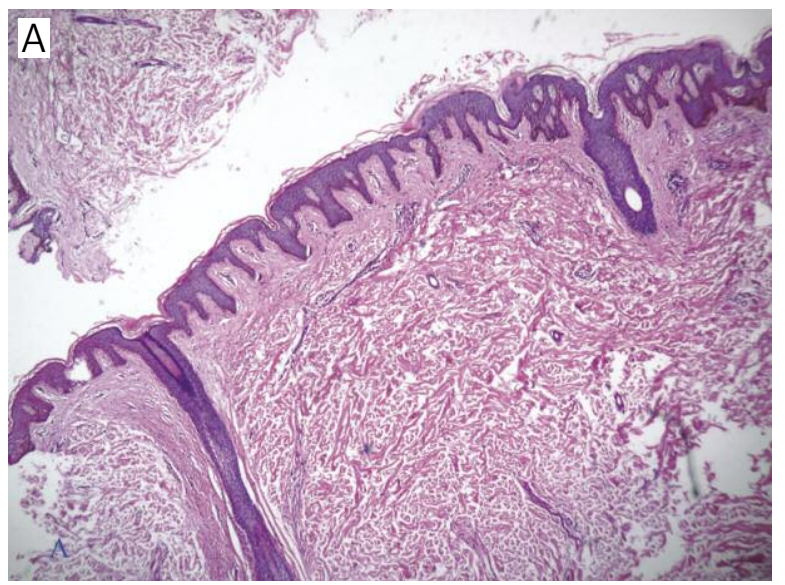

B

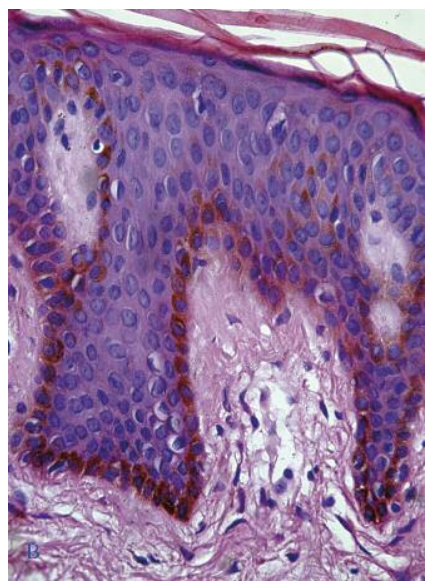

Figure 2. A - Rete ridges were mildly elongated. No melanocyte nests were noted $(H+E, 100 x)$. B - Remarkable basilar hyperpigmentation (pigmented eye line sign) without melanocytic hyperplasia. No junctional nests of melanocytes or melanocytic hyperplasia were appreciated. The dermis was almost normal except foci melanophages in the papillary dermis $(H+E, 400 x)$

autoantibodies, Glutamic-pyruvic transaminase, Alanine transaminase, blood urea nitrogen, creatinine and blood glucose. Tumor immunology markers of AFP, CEA, CA19-9, CA72-4, CA21-1, NSE and PSA were all within normal limits. He did not accept depigmentation treatment and was still in the follow-up.

\section{Case 2}

This 67-year-old male was the father of the previous patient. He noticed spotted and terrazzo-like pigmented macules on the hands about 40 years prior to this consultation. And it progressed to affect the whole arms 8 years after its initiation. He excluded a history of haphazard chemical exposure and an abnormal drug administration. This patient was otherwise in a good health condition except high blood pressure that began about 10 yeas ago. Among his family members, only one of his 3 sons had similar onset of such a skin problem. His parents, 2 sisters, 1 brother and the other 2 sons were all exempted from this condition. Physical examination reveals numerous discrete spots or macules that share the character with that of his son, except that the lesion was less generalized to both arms. Skin biopsy from another hospital noted a black eyeliner-like hyperpigmentation in the basal cell layer. Melanocytes were in 


\section{Diffuse hyperpigmentation \\ $r$}

Distinctive evidence for endocrine

or metabolic abnormalities

$(-) \sqrt{\overleftarrow{\text { In the case of a coincidence }}}$

Distinctive evidence for infections

$(-) \sqrt{\overleftarrow{\text { In the case of a coincidence }}}$

Distinctive evidence for malnutrition

$(-) \sqrt{\overleftarrow{\text { In the case of a coincidence }}}$

Distinctive evidence for tumors

$$
(-) \sqrt{\overleftarrow{\text { In the case of a coincidence }}}
$$

Distinctive evidence for drug-related or exogenous factors

$$
( - ) \longdiv { \overleftarrow { \text { In the case of a coincidence } } }
$$

Distinctive evidence for a syndrome

$$
( - ) \longdiv { \text { In the case of a coincidence } }
$$

Distinctive evidence for autoimmune diseases

$$
(-) \sqrt{\overleftarrow{\text { In the case of a coincidence }}}
$$

Distinctive evidence for other systemic disorders

$$
(-) \sqrt{\overleftarrow{\text { In the case of a coincidence }}}
$$

\section{I try my best, but still could not find contributory signs of systemic involvement}

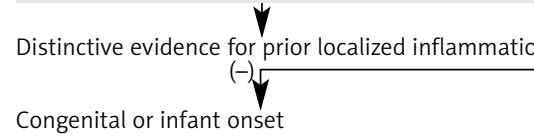

$(-\sqrt{2}$

Reticulated hyperpigmentation

$(-) \sqrt{1}$
Addison's disease, asthma melanodermica, Gaucher disease, porphyria cutanea tarda, familial glucocorticoid deficiency, lysosomal storage disease, adrenoleukodystrophy,

(+) AIDS, malaria, visceral leishmaniasis, Whipple's disease, or tinea versicolor?

(+) $\mathrm{B}_{12}$ deficiency, malabsorption, pellagra, protein deficiency, scurvy, vitamin A deficiency, or fanconi anemia? Carcinoid syndrome, ectopic ACTH-secreting tumor, metastatic melanoma, or Hodgkin's
lymphoma?

$(+)$

Phytophotodermatitis, metal deposition, toxic oil syndrome, radiotherapy, eosinophilia myalgia syndrome, tanning, phototherapy of neonatal jaundice, or variable drugs (omitted) induced?

(+) Cronkhite-Canada syndrome, Berlin syndrome, Costello syndrome, Haber syndrome, Elejalde syndrome, Werner's syndrome, Carlton Biggs syndrome, Felty's syndrome, LAM syndrome, LEOPARD syndrome, Nelson's syndrome, Hurler's syndromes, eosinophiliamyalgia syndrome, Peutz-Jegher syndrome, dyskeratosis congenita, Proteus syndrome, Winchester syndrome, Noonan's syndrome, Schmidt's syndrome, Ruvalcaba-Myhre syndrome, or Degos-Touraine syndrome?

(+) Lupus erythematous, polyglandular autoimmune syndrome type II, Still's disease or scleroderma?

(+) Primary biliary cirrhosis, renal disease, diabetes, or tuberous sclerosis?

(t) or post-inflammatory pigmentation

(+) Epidermolysis bullosa simplex with mottled pigmentation, congenital diffuse melanosis, incontinentia pigmenti, neurofibromatosis type I, transient neonatal hypermelanosis, carbon baby syndrome, tor xeroderma pigmentosum

(+) Franceschetti-Jadassohn-Naegeli syndrome, dermatopathia pigmentosa reticularis Rothmund-Thomson syndrome, confluent and reticulated papillomatosis, lichen planus, Dowling-Degos disease or Cantu's syndrome

Whorled, Blaschkoid, twin spotting and checkboard pattern lesions

Phylloid pigmented macules

Progressive cribriform and zosteriform hyperpigmentation, McCune-Albright syndrome familial progressive hyperpigmentation (partial), linear and whorled nevoid hypermelanosis, phakomatosis pigmentovascularis, or mosaicism

(+) Phylloid hypermelanosis

Colocalization with hypopigmentation

$\sqrt[(-)]{1}$

(+) Dyschromatosis universalis hereditaria, or familial progressive hyperpigmentation with white dots (in my opinion)

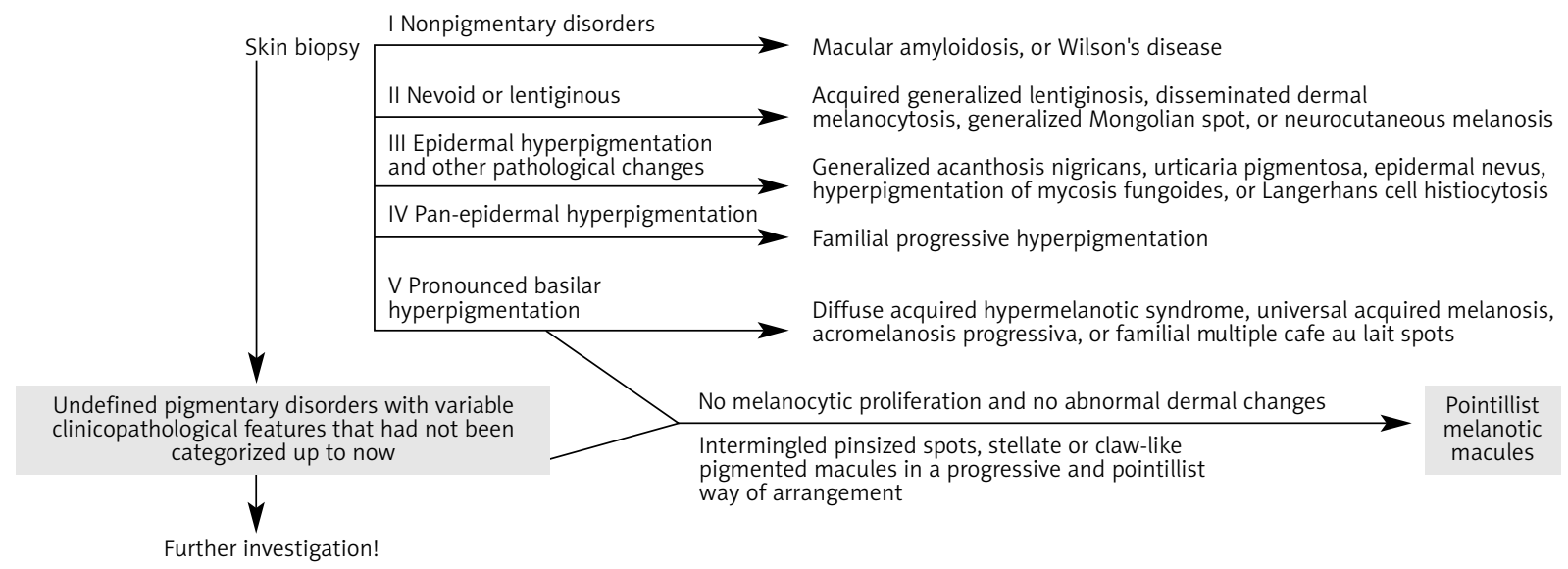

Figure 3. A stepwise approach to the diagnosis of diffuse hyperpigmentation 
normal number and morphology. There was no lentiginous elongation of the rete ridges. The dermis was normal.

The progressive nature of the lesions, late onset of the disease, benign prognosis, spotting and stellate black macules together with the prominent basilar hyperpigmentation made the preexisting dermatological terminology incapable of establishing an appropriate diagnosis. We thus proposed pointillist melanotic macules (PMM) for our patients.

\section{Discussion}

Pigmentary skin disorder perhaps has the most kaleidoscopic demonstrations benefitting from its visibility to our eyes. Some lesions are characterized to be agminated, linear, segmental, reticular, zosteriform, or following Blaschko's lines. These unique morphological features exclusively make themselves to be distinctive from each other, and thus, are conducive to a proper diagnosis. Dermatologists never slow down their steps to explore secluded regularities of pigmentary lesions, a recent report of phylloid hypomelanosis had set a good example for us notwithstanding that the authors, at the beginning, were not confident whether it was a new entity or not [1]. A characteristic configuration of a pigmentary lesion helps clinicians to approach a correct diagnosis and facilitates a sound categorization or further exploration in a uniform manner. The moment our patients confronted us, descriptive words prompted instantly were variegate, discrete, mottling, notchy and so on. However, these terms are rather ambiguous, and always fail to remodel genuine morphology of the lesion if simultaneously there is no clinical picture. Some researchers evaded such a problem simply by using 'mottling' or 'irregular' when an uncommon bizarre lesion presented [2, 3]. Instead of this, culprit locus to such a pigmentary disorder had been mapped to a certain chromosome beforehand [4-6]. Without a detailed description of the lesions, didactic value of these manuscripts will definitely be discounted by the upcoming readers, and even adds confusion to it though actually this could have been avoided at the beginning.

The disease in the patients seems to be restricted to the skin and is not associated with systemic disorders. Although genetically both originated in first degree relatives of the same pedigree, we cannot assert its type of heredity at present due to limited cases in this family. Lesions in our patient were strikingly different from those of the one previously described. Apart from thousands of pin-sized black, round and oval dots, a large majority of the lesions were stellate or claw-like pigmented macules that differ from each other in shape and size. However, as a whole, these discrete lesions were interspersed regularly, and this way of distribution prompted us a picturesque pointillist artwork for their similarities: weird on separate inspection, but well-arranged if seen at a certain distance. Pathological changes in our case were nonspecific, includ- ing a remarkable basilar hyperpigmentation without melanocytic hyperplasia, mildly-elongated rete ridges and foci melanophages in the papillary dermis. It seems that dermatological nomenclatures at present were all inappropriate to our patients before the nomenclature of PMM was proposed by us. We believe such a lexicon embraces an unprecedented clinicopathological profile in similar cases as we presented herein.

Generalized hyperpigmentation may occur as a primary disorder or a secondary feature of inborn errors of metabolism and inflammatory diseases, as well as endocrinological disorders [7, 8]. The absence of certain medical history, associated clinical findings, abnormal laboratory values, and other significant skin findings exclude PMM from most diffuse hypermelanosis under the guidance of a flowchart (Figure 3). Familial multiple cafe au lait spots (FMCALS) represent a rare, autosomal dominant pigmentary disorder characterized by multiple café au lait spots, which appear at infancy or earlier childhood as sharply defined, round to oval, homogenous macules or patches [5, 9]. Stellate or claw-like macules as presented in our cases have never been documented. In addition, it preferentially affects unexposed area mostly on the trunk. The neck and extremities are not affected. These features made FMCALS inappropriate for our patients. Mucosal melanotic macules (MMM) was another skin disorder to be differentiated. Mucosal melanotic macules is accentuated by asymmetry, irregular borders, multifocality, variegated pigmentary patterns, and large size macules [10-12]. Histopathological findings include mild acanthosis and hyperpigmentation of basal keratinocytes with scattered melanophages in the dermis. The similarity between PMM and MMM is apparent except their involving sites, which has led to the conjecture that PMM is probably the disseminated cutaneous counterpart of MMM.

Pointillist melanotic macules should be considered when the following criteria are met: 1) Numerous pigmented spots and stellate or claw-like macules disseminate over a large area of the skin; 2) Increased basilar hyperpigmentation (positive 'black eyeline sign') without melanocyte hyperplasia; 3)The dermis is otherwise normal except macrophages; 4) The last prerequisite is a negative sign of systemic involvement and dermatological manifestation indicative of well-established diagnosis.

\section{References}

1. Happle R. Phylloid hypermelanosis: an unusual frm of pigmentary mosaicism. Dermatology 2010; 220: 169-72.

2. Verbov J. Hereditary diffuse hyperpigmentation. Clin Exp Dermatol 1980; 5: 227-34.

3. Betts CM, Bardazzi F, Fanti PA, et al. Progressive hyperpigmentation: case report with a clinical, histological, and ultrastructural investigation. Dermatology 1994; 189: 384-91.

4. Zhang C, Deng Y, Chen X, et al. Linkage of a locus determining familial progressive hyperpigmentation (FPH) to chromosome 19p13.1-pter in a Chinese family. Eur I Dermatol 2006; 16: 246-50. 
5. Arnsmeier SL, Riccardi VM, Paller AS. Familial multiple cafe au lait spots. Arch Dermatol 1994; 130: 1425-6.

6. Cardinali G, Kovacs D, Giglio MD, et al. A kindred with familial progressive hyperpigmentation-like disorder: implication of fibroblast-derived growth factors in pigmentation. Eur J Dermatol 2009; 19: 469-73.

7. Greipp PR. Hyperpigmentation syndromes (diffuse hypermelanosis). Arch Intern Med 1978; 138: 356-7.

8. Uyttendaele H, Koss T, Bagheri B, et al. Generalized acanthosis nigricans in an otherwise healthy young child. Pediatr Dermatol 2003; 20: 254-6.

9. Cohen JB, Janniger CK, Schwartz RA. Cafe-au-lait spots. Cutis 2000; 66: 22-4.

10. Meleti M, Vescovi P, Mooi WJ, Van Der Waal I. Pigmented lesions of the oral mucosa and perioral tissues: a flow-chart for the diagnosis and some recommendations for the management. Oral Surg Oral Med Oral Pathol Oral Radiol Endod 2008; 105: 606-16.

11. Lenane P, Keane CO, Connell BO, et al. Genital melanotic macules: clinical, histologic, immunohistochemical, and ultrastructural features. I Am Acad Dermatol 2000; 42: 640-4.

12. Kaugars GE, Heise AP, Riley WT, et al Oral melanotic macules. A review of 353 cases. Oral Surg Oral Med Oral Pathol 1993; 76: 59-61. 